Braz J Med Biol Res, October 2011, Volume 44(10) 1054-1059

doi: 10.1590/S0100-879X2011007500118

\section{Reduced hippocampal GABAergic function in Wistar audiogenic rats}

L.E. Drumond, C. Kushmerick, P.A.M. Guidine, M.C. Doretto, M.F.D. Moraes and A.R. Massensini

The Brazilian Journal of Medical and Biological Research is partially financed by

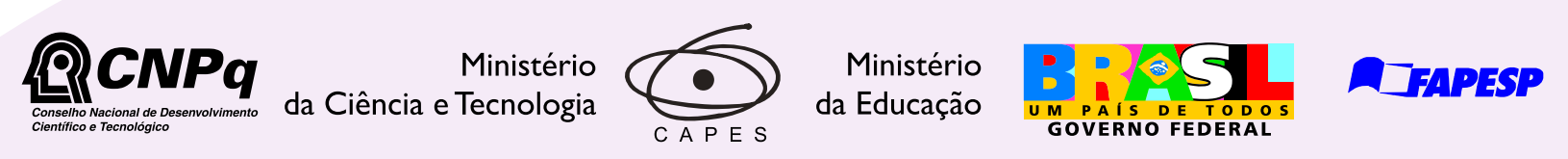

Institutional Sponsors
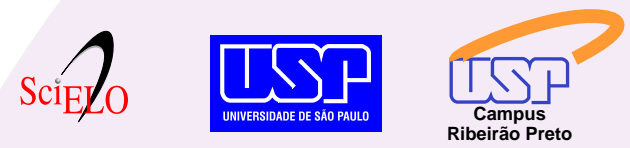

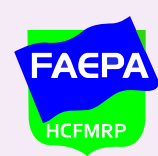

Ф SHIMADZU

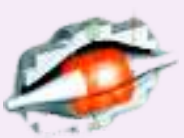

Explore High - Performance MS Orbitrap Technology In Proteomics \& Metabolomics

$\underset{\text { analitica }}{\text { analiticaweb.com.br }}$ SCIENTIFIC 


\title{
Reduced hippocampal GABAergic function in Wistar audiogenic rats
}

\author{
L.E. Drumond ${ }^{1}$, C. Kushmerick ${ }^{2}$, P.A.M. Guidine ${ }^{1}$, M.C. Doretto ${ }^{1}$, \\ M.F.D. Moraes ${ }^{1}$ and A.R. Massensini ${ }^{1}$
}

${ }^{1}$ Núcleo de Neurociências, ${ }^{2}$ Laboratório de Eletrofisiologia Celular, Departamento de Fisiologia e Biofísica, Instituto de Ciências Biológicas, Universidade Federal de Minas Gerais, Belo Horizonte, MG, Brasil

\begin{abstract}
Epilepsy is a neurological disorder associated with excitatory and inhibitory imbalance within the underlying neural network. This study evaluated inhibitory $y$-amino-butyric acid (GABA)ergic modulation in the CA1 region of the hippocampus of male Wistar rats and Wistar audiogenic rats (aged $90 \pm 3$ days), a strain of inbred animals susceptible to audiogenic seizures. Field excitatory postsynaptic potentials and population spike complexes in response to Schaffer collateral fiber stimulation were recorded in hippocampal slices before and during application of picrotoxin ( $50 \mu \mathrm{M}, 60 \mathrm{~min}$ ), a GABA antagonist, and the size of the population spike was quantified by measuring its amplitude and slope. In control audiogenic-resistant Wistar rats $(\mathrm{N}=9)$, picrotoxin significantly increased both the amplitude of the population spike by $51 \pm 19 \%$ and its maximum slope by $73 \pm 21 \%$. In contrast, in slices from Wistar audiogenic rats $(\mathrm{N}=6)$, picrotoxin caused no statistically significant change in population spike amplitude $(33 \pm 46 \%)$ or slope $(11 \pm 29 \%)$. Data are reported as means \pm SEM. This result indicates a functional reduction of GABAergic neurotransmission in hippocampal slices from Wistar audiogenic rats.
\end{abstract}

Key words: Epilepsy; Extracellular recording; GABA; Hippocampus; Wistar audiogenic rats; Picrotoxin

\section{Introduction}

Epilepsy is a serious neurological condition that affects approximately 50 million people worldwide (1). It is characterized by recurrent seizures related to excessive or synchronous neural activity in the brain (2). At the cellular level, alterations that could underlie epileptic brain hyperactivity include changes in the membrane passive properties or in voltage gated channels or alternations in synaptic connections that increase excitability (3-7).

The acute brain slice preparation provides a useful electrophysiological model to study cellular aspects of epilepsy in animal models because it allows control of the extracellular medium and maintains local neuronal circuits intact $(8,9)$. Here, we examined the role of $y$-amino-butyric acid (GABA)ergic inhibition in hippocampal slices from Wistar audiogenic rats, a strain developed by selected inbreeding for susceptibility to audiogenic seizures (10-12). In these rats, a single high-intensity acoustic stimulation induces generalized tonic-clonic convulsions followed by clonic spasms. The onset of audiogenic seizures in these animals primarily reflects brain stem-dependent mechanisms since they can be evoked after forebrain removal
(13). However, repetitive chronic stimulation provokes a change in the EEG and in seizure phenotype suggestive of selective recruitment of limbic structures including the hippocampus and amygdala (11,14-17). This interchange of brainstem/forebrain circuitry in the phenotype of audiogenic seizures could be explained by a generalized seizure predisposition in the brain of Wistar audiogenic rats. Consistent with this hypothesis, these rats show abnormal sensitivity to a variety of seizure-provoking stimuli including maximum convulsive electroshock, pentylenetetrazole and pilocarpine (18), suggesting inherited low threshold epileptogenic neural circuits.

GABA, acting through specific receptors, mediates inhibition throughout the mammalian brain (19-21). GABAergic neurons are often local circuit inhibitory cells that exhibit diverse morphological, biochemical, molecular, and synaptic properties (22,23). For these cells, the anatomical location of the GABAergic synapses on the postsynaptic cell in large part determines the functional impact of interneuronal activity on the target neuron. Thus, interneurons targeting perisomatic regions

Correspondence: P.A.M. Guidine, Núcleo de Neurociências, Departamento de Fisiologia e Biofísica, ICB, UFMG, Av. Antônio Carlos, 6627, 31270-901 Belo Horizonte, MG, Brasil. Fax: +55-31-3409-2924. E-mail: maiaguidine@gmail.com

Received December 30, 2010. Accepted August 25, 2011. Available online September 9, 2011. Published October $10,2011$. 
are strategically placed to modulate somatic action potential generation $(24,25)$, whereas those terminating more distally may prevent dendritic action potentials (25), modulate the induction of glutamatergic synaptic plasticity (26), or gate the transfer of information along the somatodendritic axis (27). Pharmacological block of $\mathrm{GABA}_{A}$ receptors in vivo causes seizure and epileptic-like EEG signals (28) and epileptiform electrical discharges in in vitro slice preparations (29). Therefore, alterations in GABAergic inhibition could contribute towards the hyperexcitability and hypersynchronism typically found in epilepsies $(19,21,30)$.

Previous studies have demonstrated a reduction in GABA receptor-mediated current density in cultured hippocampal neurons obtained from Wistar audiogenic rats (7), suggesting a reduction in GABAergic inhibition in the hippocampus of these animals. To test this hypothesis we evaluated the influence of $\mathrm{GABA}_{A}$ receptors on neurotransmission in the CA1 region of hippocampus slices from Wistar audiogenic rats compared to slices obtained from control audiogenic-resistant Wistar rats.

\section{Material and Methods}

\section{Animals}

Male Wistar rats (13 weeks old) and male Wistar audiogenic rats (13 weeks old) were supplied by the CEBIO-ICB-UFMG vivarium. The animals had free access to food and water and were housed in a temperaturecontrolled environment under a 12-h light-dark cycle until the start of the procedure. Efforts were made to avoid any unnecessary distress to the animals, in accordance with $\mathrm{NIH}$ guidelines for the care and use of animals and with approved animal protocols from the Institutional Animal Care and Use Committees of Universidade Federal de Minas Gerais (Protocol No. 131/05).

Audiogenic seizure sensitivity and severity were evaluated using the severity index (SI) developed by Garcia-Cairasco et al. (15). This index ranges from SI $=0$ (no seizures, audiogenic-resistant rats) to $\mathrm{SI}=1$ (generalized tonic-clonic seizures with fore and hind limb hyperextension: highly susceptible rats). Animals were screened with sound from a buzzing doorbell (120 dB SPL, $60 \mathrm{~s}$ ). Three screening trials were performed with a minimum interval of $48 \mathrm{~h}$ between each trial. Rats were excluded from the audiogenic-resistant control group if they presented a severity index $>0$ in any of the three trials. Rats were excluded from the audiogenic group if they failed to present a severity index of 0.85 or greater in at least two of three trials.

\section{Electrophysiology}

The animals were decapitated 1 week after the end of the screening test and their brains were quickly removed from the skull. Coronal slices ( $400-\mu m$ thick) were prepared with a Vibratome (Leica VT 1000P, Technical Products International, USA) in ice-cold low-calcium artificial cerebrospinal fluid (aCSF) containing $125 \mathrm{mM} \mathrm{NaCl}, 2.5 \mathrm{mM}$ $\mathrm{KCl}, 3.0 \mathrm{mM} \mathrm{MgCl}_{2}, 0.1 \mathrm{mM} \mathrm{CaCl}_{2}, 25 \mathrm{mM}_{\text {glucose, }} 25 \mathrm{mM}$ $\mathrm{NaCO}_{3}, 1.25 \mathrm{mM} \mathrm{NaH}_{2} \mathrm{PO}_{4}$ bubbled with $95 \% \mathrm{O}_{2}$ and $5 \%$ $\mathrm{CO}_{2}$ to $\mathrm{pH}$ 7.4. The slices were allowed to recover at room temperature for at least $60 \mathrm{~min}$ before being transferred individually to a submerged recording chamber, which was superfused (1-2 $\mathrm{mL} / \mathrm{min}$ ) with oxygenated normal aCSF at room temperature $\left(25^{\circ} \mathrm{C}\right)$. The normal aCSF had the same composition as the low-calcium aCSF, except for the concentrations of $1.0 \mathrm{mM} \mathrm{MgCl}_{2}$ and $2.0 \mathrm{mM} \mathrm{CaCl}_{2}$ in the normal aCSF. The recording chamber was mounted on the stage of an upright microscope Axioskop (Zeiss, Germany).

Schaffer collateral fibers were electrically stimulated once every $10 \mathrm{~s}$ using monophasic rectangular pulses of $100-\mu$ s duration at stimulus intensities of $50-80 \mathrm{~V}$ through a bipolar platinum microelectrode placed on the stratum radiatum. Field potentials were recorded in the CA1 pyramidal cell region with microelectrodes pulled from borosilicate glass capillaries (World Precision Instruments, TW150F-3, USA) with a resistance of 0.5-1 M $\Omega$ when filled with standard aCSF. For each experiment, an input/output curve for stimulus vs amplitude of the population spike (PS) was generated. The stimulation intensity was then set to the lowest voltage that gave a maximum response and was not adjusted further for the remainder of the experiment. Stimuli were applied every $10 \mathrm{~s}$ and field excitatory postsynaptic potential-PS (EPSP-PS) complexes were recorded throughout the remainder of the experiment. After the response had stabilized, we recorded for $10 \mathrm{~min}$ in control aCSF to establish the baseline amplitude of the population spike. To assure that the signal was a postsynaptic response, we then recorded in 0-Ca aCSF containing no added $\mathrm{CaCl}_{2}$ (substituted with equimolar $\mathrm{MgCl}_{2}$ ) and 2 mM EGTA for $5 \mathrm{~min}$. The slice was then superfused with oxygenated normal aCSF and recordings were continued for $30 \mathrm{~min}$ to allow for recovery from 0-Ca aCSF, followed by the addition of $50 \mu \mathrm{M}$ picrotoxin (Research Biochemicals International, USA), a $\mathrm{GABA}_{\mathrm{A}}$ receptor antagonist, to block fast GABAergic transmission. We recorded in the presence of picrotoxin for a further $60 \mathrm{~min}$. Field potential responses were amplified and filtered (band pass between $1 \mathrm{~Hz}$ and $3 \mathrm{KHz}$ ) with a CyberAmp 380 (Axon Instruments, USA), digitized at $25 \mathrm{KHz}$ using a National Instruments A/D board and stored in a personal computer running the acquisition program WinWCP 4.2 (Strathclyde Electrophysiology Software, University of Strathclyde, Glasgow, Scotland).

\section{Waveform analysis}

Extracellular EPSP-PS waveforms were analyzed using custom written software within Igor Pro 6 (Wave- 
metrics, USA). The program calculated the amplitude of the PS, its latency, and the maximum negative slope during the descending phase. Data were imported into Igor and visually inspected using Neuromatic (Version 2.00, kindly provided by Dr. J. Rothman, University College of London, London). Data were smoothed using 50 passes of the built-in binomial smoothing function of Igor. The EPSP-PS complex was identified manually as a triphasic waveform with a negative peak occurring 10-15 ms after the stimulation artifact. The operator placed cursors on the descending and ascending portions of the curve on either side of its negative peak. The program then determined the beginning and end points of the PS. The beginning point of the PS was either a local minimum in dv/dt or the point at which dv/dt crossed zero, whichever was found first going backward in time from the left-hand cursor. The end-point of the population spike was the first point after the negative peak for which a line drawn from the beginning point was tangent to the waveform at that point. The amplitude of the PS was the vertical distance between this line and the negative peak. The slope of the EPSPPS complex was measured by linear regression over 20 data points centered at the local minimum in $\mathrm{dv} / \mathrm{dt}$ that occurred during the PS downstroke and PS latency was measured relative to the stimulus artifact. To analyze a sequence of EPSP-PS waveforms, the program optionally permitted automatic repositioning of the cursors to compensate for drift in latency during an experiment. When this option was enabled, after each PS was processed, the left hand cursor was automatically moved to the point of maximum dv/dt during the downward stroke of the PS and the right hand cursor was moved to $1 / 3$ the distance from the negative peak to the endpoint of the PS, and these initial cursor values were used to identify the next $P S$ in the sequence.

\section{Statistical analysis}

Data were obtained from 9 slices from 7 audiogenicresistant Wistar rats and 6 slices from 6 Wistar audiogenic rats. In one additional experiment on a slice from a Wistar audiogenic rat, the PS increased $>600 \%$ upon the addition of picrotoxin. This result was declared an outlier and was excluded because the observed increase in PS was $>7$ standard deviations above the mean response observed in the remaining experiments. Group data are reported as means \pm SEM. Comparisons between groups were analyzed by one-way ANOVA and the post hoc two-tailed Student $t$-test (paired for intragroup comparisons, unpaired for intergroup comparisons).

\section{Results}

Extracellular EPSP-PS complexes were recorded from hippocampus slices and the slope and amplitude of the population spike was quantified as illustrated in Figure
1. To test if the responses recorded were postsynaptic in origin, we superfused the slices with 0-Ca aCSF and observed a strong reduction in the PS amplitude and slope with little change in the fiber volley. The average PS amplitude and slope recorded in normal aCSF, $0-\mathrm{Ca}$ aCSF, and in the presence of $50 \mu \mathrm{M}$ picrotoxin is depicted in Figure 1B for slices from control audiogenicresistant Wistar rats and in Figure $1 \mathrm{C}$ for slices from Wistar audiogenic rats. For both experimental groups, the PS amplitude and slope were strongly inhibited when superfused with 0-Ca aCSF. Upon return to normal aCSF, both parameters were larger than their initial values, but this difference was only statistically significant for the control Wistar group. Upon incubation with $50 \mu \mathrm{M}$ picrotoxin, both the amplitude and slope of the PS increased in slices from control Wistar rats, but not for slices from Wistar audiogenic rats.

To remove the substantial variability in the amplitude of the EPSP-PS complex between experiments (note the difference in vertical scale in Figure 2A), we normalized the PS amplitude and slope data for each experiment to the average value during the 10 min prior to the addition of picrotoxin, and then plotted the value of the normalized data averaged over 5-min intervals (Figure 2B,C). These data indicate that the increase in PS amplitude or slope commenced shortly after the time when picrotoxin was added to the bath, although the difference was not statistically significant for times $<20 \mathrm{~min}$. In contrast, there were no significant alterations in either PS slope or amplitude in slices from Wistar audiogenic rats. After 60-min incubation with picrotoxin, normalized PS amplitudes and slopes from the two experimental groups were significantly different from one another (amplitude: Wistar $152 \pm 15 \%$ vs Wistar audiogenic rats $99 \pm 17 \%$, $\mathrm{P} \leq 0.05$; slope: Wistar $194 \pm 28 \%$ vs Wistar audiogenic rats $87 \pm 17 \%, P \leq 0.05)$.

\section{Discussion}

Wistar audiogenic rats have been inbred for more than 50 generations using a selection criterion based on the severity and phenotype of the seizure (13). They have an inherited predisposition to seizures (13), representing a useful animal model for the study of the pathophysiology of epilepsy. We observed a marked increase in PS amplitude and slope in hippocampal slices from control Wistar rats after application of picrotoxin but not in slices from Wistar audiogenic rats. The PS, measured in the pyramidal layer of the CA1 region, is the extracellular potential caused by action potentials in these cells. Thus, our data suggest that GABAergic inhibition of CA1 action potential generation is reduced in Wistar audiogenic rats.

Picrotoxin is a noncompetitive antagonist that reduces $\mathrm{Cl}^{-}$current through $\mathrm{GABA}_{A}$ receptors (31). The concentration of picrotoxin we applied $(50 \mu \mathrm{M})$ is 3.6 times the 
A
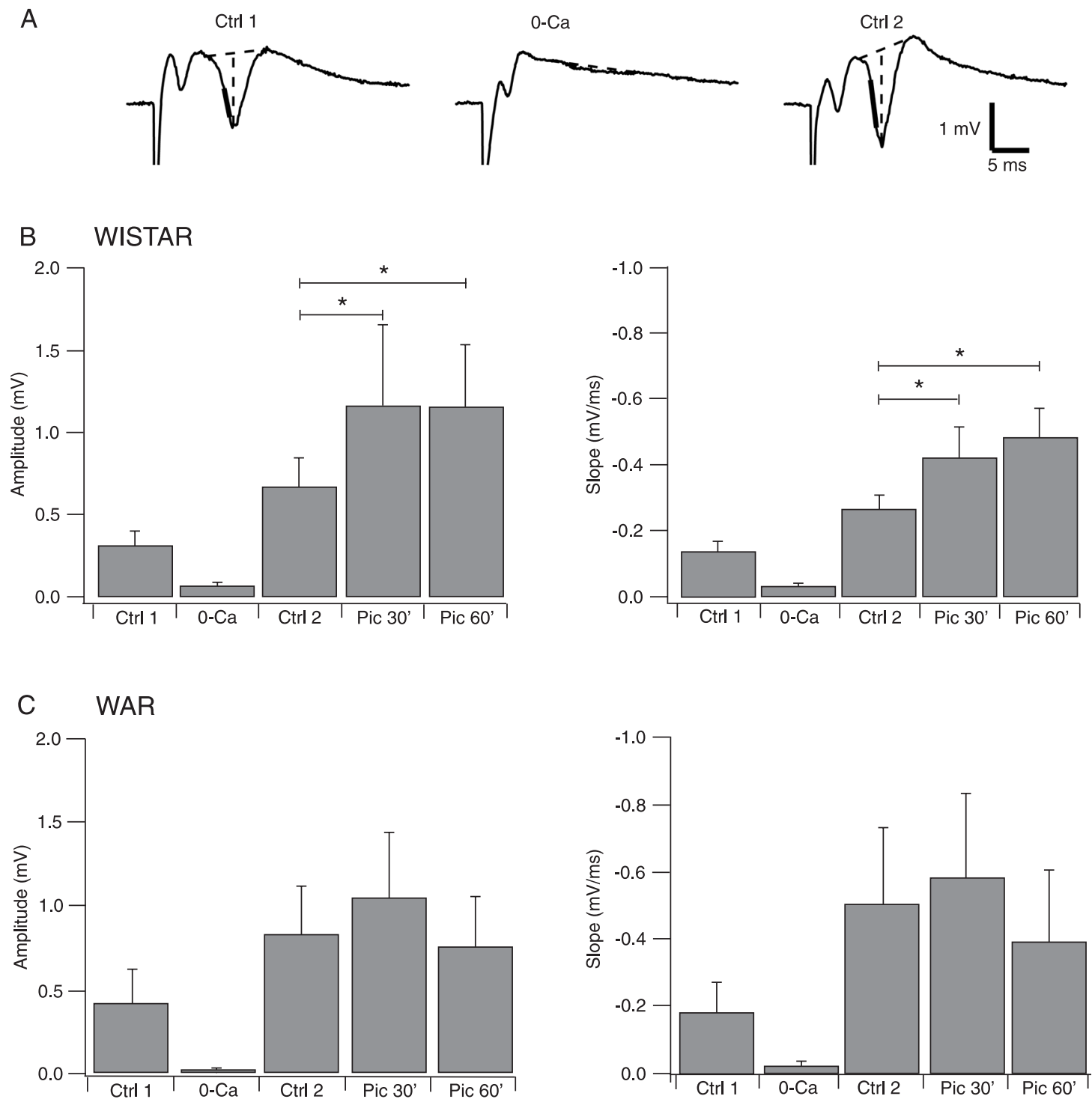

Figure 1. Ca-dependent EPSP-PS complexes recorded in the CA1 region of the hippocampus of Wistar audiogenic rats have reduced sensitivity to picrotoxin $(50 \mu \mathrm{M})$. A, Example waveforms obtained in the CA1 pyramidal layer of a hippocampal slice from a Wistar audiogenic rat in control artificial cerebrospinal fluid (aCSF), in 0-Ca aCSF containing $2 \mathrm{mM}$ EGTA, and upon return to normal aCSF. The waveforms shown are the average of 6 responses in each experimental condition. The broken lines illustrate the method used to analyze the amplitude of the population spike. The thick black line indicates the region used to measure the maximum slope. See Methods for the details of waveform analysis. $B$ and $C$, Summary of the amplitude and slope of the population spike obtained in slices from control audiogenic-resistant Wistar rats $(B$; WISTAR, $\mathrm{N}=9$ slices from 7 animals) and from Wistar audiogenic rats $(C ;$ WAR, $\mathrm{N}=6$ slices from 6 animals), in control aCSF (Ctrl 1), in 0-Ca aCSF (0-Ca), upon return to normal aCSF (Ctrl 2), after 30-min treatment with picrotoxin (Pic 30'), and after 60-min treatment with picrotoxin (Pic 60'). Error bars are 1 SEM. *P $\leq 0.05$ (one-way ANOVA, with two-tailed Student $t$-test, paired for intragroup comparisons, unpaired for intergroup comparisons).

EC50 for inhibition of $\mathrm{GABA}_{A}$ receptor current in cultured hippocampal neurons (32). We therefore expect to have blocked a large portion of $\mathrm{GABA}_{A}$-mediated inhibition in our experiments. In slices from control audiogenicresistant Wistar rats, we observed a statistically significant increase in PS size 20 min after application of picrotoxin, and the response reached a plateau after $40 \mathrm{~min}$. Probably, the time necessary for the diffusion of picrotoxin within the slice accounts for only a small part of this latency. For comparison, we note that when we applied $0-\mathrm{Ca}$ 
aCSF containing $2 \mathrm{mM}$ EGTA to the slice the size of the PS fell with a time constant of 2-4 min (data not shown). A second factor that may explain the slow onset of the effect of picrotoxin is the fact that we used a stimulus intensity that generated maximal PS amplitude. This choice of stimulation protocol may have required a block of a substantial portion of $\mathrm{GABA}_{A}$ receptors by picrotoxin before producing a significant change in PS.

Our results extend previous findings of reduced GABAergic current density in cultured hippocampal neurons from Wistar audiogenic rats (7). In addition to a reduction in inhibitory currents, these investigators also reported changes in resting potential, membrane input resistance, and potassium current density that all favor enhanced excitability. Similar findings have been reported in other animal models of epilepsy. The genetically epilepsy-prone rat (GEPR), an audiogenic strain similar to Wistar audiogenic rats, exhibited reduced sensitivity to exogenous GABA in auditory brainstem circuits (33). In addition, electrophysiological studies of hippocampal slices from adult GEPR rats suggest several features leading to increased excitability, including a reduced $\mathrm{GABA}_{A}$-mediated inhibition, increased input impedance, reduced spike frequency adaptation, and increased paired-pulse facilitation (34-36). Interestingly, these investigators have reported significant differences in the cellular alterations observed in the CA1 and $\mathrm{CA} 3$ regions of the GEPR hippocampus, indicating that epileptic hyperactivity at the cellular level may be highly dependent on the specific brain region examined. Hippocampal CA1 neurons have also been studied in rats in which recurring epileptic seizures were induced with pilocarpine. These studies indicate a higher input impedance, reduced resting potential and reduced spike frequency adaptation in neurons from epileptic animals (3). Thus, studies on hippocampal neurons from different animal models of epilepsy indicate two possible sources of neuronal hyperexcitability - changes in intrinsic neuronal properties and reduced GABAergic inhibition. Our results suggest that the latter is important in the hippocampus of Wistar audiogenic rats.

\section{Acknowledgments}

We are grateful to Adriano Jesus da Silva and Jaderson Costa da Costa for useful discussions of field potential recording techniques, to José Eustáquio Oliveira for technical assistance, and to the Brazilian agencies CNPq, FAPEMIG, CAPES, and PRPq/UFMG for financial support.
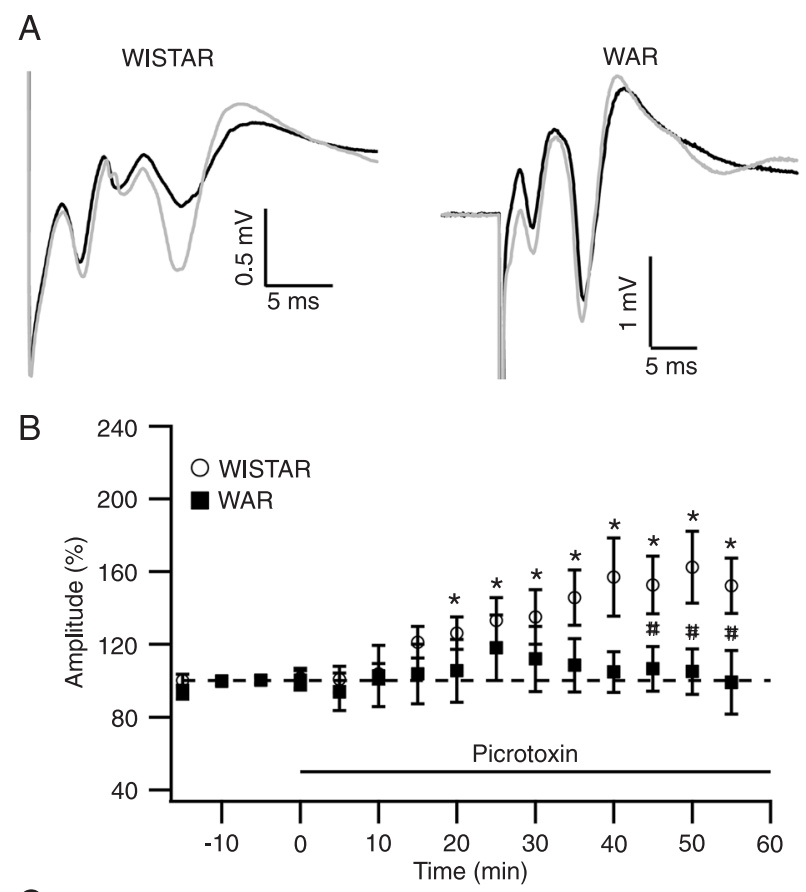

C

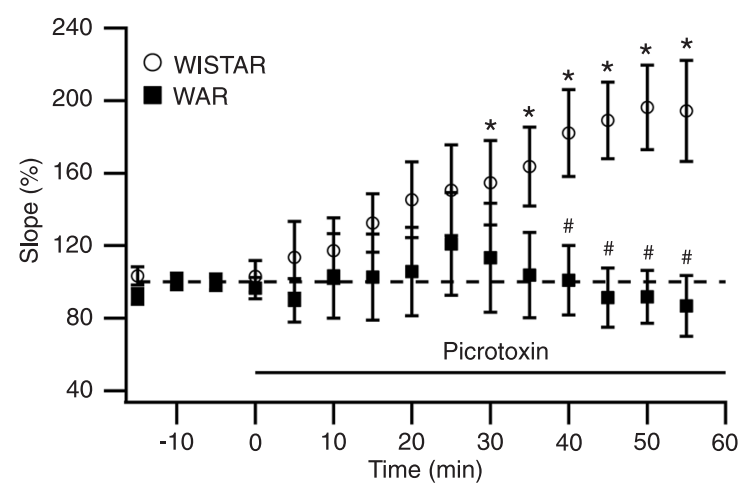

Figure 2. Picrotoxin $(50 \mu \mathrm{M})$ causes a time-dependent increase in population spike (PS) amplitude and slope in control Wistar rats that is absent in Wistar audiogenic rats. $A$, Example of hippocampal CA1 excitatory postsynaptic potential-PS (EPSP-PS) complexes for slices from a control Wistar rat (WISTAR) or a Wistar audiogenic rat (WAR). Black traces, average of 30 waveforms recorded during the last $5 \mathrm{~min}$ before picrotoxin application. Gray traces, average of 30 waveforms recorded between 55 and $60 \mathrm{~min}$ of picrotoxin application. $B$ and $C$, Time course of the normalized PS amplitude $(B)$ and PS slope $(C)$ during application of picrotoxin. The broken line is drawn at $100 \%$. ${ }^{*} \mathrm{P} \leq 0.05$, significantly different from $100 \%$; $P \leq 0.05$, compared to Wistar (oneway ANOVA, with two-tailed Student $t$-test, paired for intragroup comparisons, unpaired for intergroup comparisons). 


\section{References}

1. Sander JW. The epidemiology of epilepsy revisited. Curr Opin Neurol 2003; 16: 165-170.

2. Fisher RS, van Emde Boas W, Blume W, Elger C, Genton P, Lee $P$, et al. Epileptic seizures and epilepsy: definitions proposed by the International League Against Epilepsy (ILAE) and the International Bureau for Epilepsy (IBE). Epilepsia 2005; 46: 470-472.

3. Bravo-Martinez J, Delgado-Coello B, Garcia DE, Mas-Oliva $\mathrm{J}$. Analysis of plasma membrane $\mathrm{Ca}^{2+}$-ATPase gene expression during epileptogenesis employing single hippocampal CA1 neurons. Exp Biol Med 2011; 236: 409-417.

4. Dichter MA, Ayala GF. Cellular mechanisms of epilepsy: a status report. Science 1987; 237: 157-164.

5. Dudek FE, Sutula TP. Epileptogenesis in the dentate gyrus: a critical perspective. Prog Brain Res 2007; 163: 755-773.

6. Escayg A, Goldin AL. Sodium channel SCN1A and epilepsy: mutations and mechanisms. Epilepsia 2010; 51: 16501658.

7. Mesquita F Jr, Aguiar JF, Oliveira JA, Garcia-Cairasco N, Varanda WA. Electrophysiological properties of cultured hippocampal neurons from Wistar Audiogenic Rats. Brain Res Bull 2005; 65: 177-183.

8. Dingledine R, Dodd J, Kelly JS. The in vitro brain slice as a useful neurophysiological preparation for intracellular recording. J Neurosci Methods 1980; 2: 323-362.

9. Schurr A, West CA, Rigor BM. Electrophysiology of energy metabolism and neuronal function in the hippocampal slice preparation. J Neurosci Methods 1989; 28: 7-13.

10. Jobe PC, Picchioni AL, Chin L. Role of brain norepinephrine in audiogenic seizure in the rat. J Pharmacol Exp Ther 1973; 184: 1-10.

11. Vergnes M, Kiesmann M, Marescaux C, Depaulis A, Micheletti G, Warter JM. Kindling of audiogenic seizures in the rat. Int J Neurosci 1987; 36: 167-176.

12. Garcia-Cairasco N, Doretto MC, Prado RP, Jorge BP, Terra VC, Oliveira JA. New insights into behavioral evaluation of audiogenic seizures. A comparison of two ethological methods. Behav Brain Res 1992; 48: 49-56.

13. Garcia-Cairasco N, Sabbatini RM. Neuroethological evaluation of audiogenic seizures in hemidetelencephalated rats. Behav Brain Res 1989; 33: 65-77.

14. Naritoku DK, Mecozzi LB, Aiello MT, Faingold CL. Repetition of audiogenic seizures in genetically epilepsy-prone rats induces cortical epileptiform activity and additional seizure behaviors. Exp Neurol 1992; 115: 317-324.

15. Garcia-Cairasco N, Wakamatsu H, Oliveira JA, Gomes EL, Del Bel EA, Mello LE. Neuroethological and morphological (Neo-Timm staining) correlates of limbic recruitment during the development of audiogenic kindling in seizure susceptible Wistar rats. Epilepsy Res 1996; 26: 177-192.

16. Hirsch E, Maton B, Vergnes M, Depaulis A, Marescaux C. Positive transfer of audiogenic kindling to electrical hippocampal kindling in rats. Epilepsy Res 1992; 11: 159-166.

17. Dutra Moraes MF, Galvis-Alonso OY, Garcia-Cairasco N. Audiogenic kindling in the Wistar rat: a potential model for recruitment of limbic structures. Epilepsy Res 2000; 39: 251-259.

18. Scarlatelli-Lima AV, Magalhaes LH, Doretto MC, Moraes MF.
Assessment of the seizure susceptibility of Wistar Audiogenic rat to electroshock, pentyleneterazole and pilocarpine. Brain Res 2003; 960: 184-189.

19. Clark S, Wilson WA. Mechanisms of epileptogenesis. Adv Neurol 1999; 79: 607-630.

20. Meldrum BS. The role of glutamate in epilepsy and other CNS disorders. Neurology 1994; 44: S14-S23.

21. Schwartzkroin PA. Cellular electrophysiology of human epilepsy. Epilepsy Res 1994; 17: 185-192.

22. Freund TF, Buzsaki G. Interneurons of the hippocampus. Hippocampus 1996; 6: 347-470.

23. McBain CJ, Fisahn A. Interneurons unbound. Nat Rev Neurosci 2001; 2: 11-23.

24. Cobb SR, Buhl EH, Halasy K, Paulsen O, Somogyi P. Synchronization of neuronal activity in hippocampus by individual GABAergic interneurons. Nature 1995; 378: 75-78.

25. Miles R, Toth K, Gulyas Al, Hajos N, Freund TF. Differences between somatic and dendritic inhibition in the hippocampus. Neuron 1996; 16: 815-823.

26. Magee JC, Johnston D. A synaptically controlled, associative signal for Hebbian plasticity in hippocampal neurons. Science 1997; 275: 209-213.

27. Buzsaki G, Penttonen M, Nadasdy Z, Bragin A. Pattern and inhibition-dependent invasion of pyramidal cell dendrites by fast spikes in the hippocampus in vivo. Proc Natl Acad Sci U S A 1996; 93: 9921-9925.

28. Butuzova MV, Kitchigina VF. Repeated blockade of GABA receptors in the medial septal region induces epileptiform activity in the hippocampus. Neurosci Lett 2008; 434: 133138.

29. Knowles WD, Traub RD, Strowbridge BW. The initiation and spread of epileptiform bursts in the in vitro hippocampal slice. Neuroscience 1987; 21: 441-455.

30. Monyer H, Burnashev N, Laurie DJ, Sakmann B, Seeburg $\mathrm{PH}$. Developmental and regional expression in the rat brain and functional properties of four NMDA receptors. Neuron 1994; 12: 529-540.

31. Macdonald RL, Olsen RW. GABA $A$ receptor channels. Annu Rev Neurosci 1994; 17: 569-602.

32. Gibbs JW III, Sombati S, DeLorenzo RJ, Coulter DA. Physiological and pharmacological alterations in postsynaptic GABA(A) receptor function in a hippocampal culture model of chronic spontaneous seizures. J Neurophysiol 1997; 77: 2139-2152.

33. Faingold CL. The genetically epilepsy-prone rat. Gen Pharmacol 1988; 19: 331-338.

34. Verma-Ahuja S, Evans MS, Espinosa JA. Evidence of increased excitability in GEPR hippocampus preceding development of seizure susceptibility. Epilepsy Res 1998; 31: 161-173.

35. Verma-Ahuja S, Evans MS, Pencek TL. Evidence for decreased calcium dependent potassium conductance in hippocampal CA3 neurons of genetically epilepsy-prone rats. Epilepsy Res 1995; 22: 137-144.

36. Verma-Ahuja S, Pencek TL. Hippocampal CA1 neuronal properties in genetically epilepsy-prone rats: evidence for increased excitation. Epilepsy Res 1994; 18: 205-215. 